\title{
Article
}

\section{Numerical Modeling of Non-Newtonian Flow in Viscous Micropump}

\author{
Haifa EL-SADI, Nabil ESMAIL, and Ibrahim HASSAN \\ Department of Mechanical and Industrial Engineering Concordia University, \\ Quebec, Canada \\ (Received : August 21, 2007)
}

\begin{abstract}
Non-Newtonian fluid can be encountered in many applications of Microdevices. In this study, two-dimensional nonNewtonian simulations of viscous micropump were performed. The viscous micropump consists of a rotating cylinder located eccentrically inside a microchannel. When the cylinder rotates, a net force is transferred to the fluid due to the unequal shear stresses on the upper and lower surfaces of the cylinder, thus causing the fluid to displace. Navier Stokes equations and modified Bingham model have been used to describe the fluid flow. Parameters as viscosity and stress used in the model are based on experimental data. It was found that Reynolds number is a predominant parameter on the variation of bulk velocity as a function of eccentricity. The stress and bulk velocity decrease with increasing the eccentricity at low Reynolds number. The changes in non-Newtonian fluid structure are related to Reynolds number, eccentricity and channel height. The pumping performance of non-Newtonian fluid is increasing with global pressure gradient and decreasing with the channel height.
\end{abstract}

Key Words: Micropump / Non-Newtonian / Bingham model / Eccentricity / Bulk velocity

\section{INTRODUCTION}

Increasing efforts are being directed towards applying the technologies of microfluidic, to the development of microdevices for a wide range of applications such as medical, biological and related technologies. The main advantage of MEMS, in addition to their small size, is the fact that the manufacturing costs are remarkably lower when compared to their bigger counterparts, due to the mass fabrication methods used to produce them.

Micropumps are between the most developed of all MEMS devices, and have been executed into the mainstream. ${ }^{1,2)}$ Micropumps are imperative components for distributing fluid and samples in microanalysis system. Positive displacement pumping is the most widespread method used in micropumps, on the other hand the actuation of the reciprocating diaphragm can be achieved by different principles such as piezoelectric, pneumatic, electrostatic etc. ${ }^{3,4)}$. However, various pumping ideas were proposed to overcome the valve problem correlated with positive displacement pumps.

The novel viscous pump has attracted attention in recent years. Experimental studies were performed using a pump based on this principle at low Reynolds number. ${ }^{5)}$ The simulations for the novel viscous pump carried out are based on this principle at high Reynolds number (Newtonian). $\left.{ }^{6}\right)$ Predicting the dependence of fluid motion in open channel through the rotational cylinder and the fluid properties is still an issue receiving considerable attention in the literature. Previous studies showed numerical simulation of two and three-dimensional viscous fluid (Newtonian) and the influence of the dynamic parameters, width and other geometric. ${ }^{7,8)}$ However, many fluids demonstrate a more complicated relationship between the observed shear stress and the rate of strain as non-Newtonian fluid. There has been few studies on electro-rheological fluids whose apparent viscosity is controlled by the applied electric field have been widely researched using micro-systems ${ }^{9)}$ Therefore, much attention is given to the flow of non-Newtonian liquids in Micro-geometries. A crucial class of non-Newtonian materials exhibits a yield stress, which must be exceeded before significant deformation can occur. The models presented for such so-called viscoplastic materials included the Bingham, Herschel-Bulkley and Casson. A major review article on the subject of the pressure-driven flow of a Bingham plastic past a cylinder kept between parallel plates has appeared by E.Mitsoulis. ${ }^{10,11)}$ On the other hand, a popular approach to regularize the ideal Bingham model has been the exponential modification proposed by Papanastasiou. ${ }^{12)}$ Also, a theory for the non slip VPBL, involving a Bingham-Hooke model was published by Piau. ${ }^{13)}$ Several authors have addressed the problem of creeping flow past a rigid sphere in an unbounded regime and the problem of two rigid spheres translating in a Bingham material..$^{14,15)}$ 
Non-Newtonian fluids have many industrial applications and are of interest in MEMS devices. Non-Newtonian fluid flow effects are important for polymeric liquids, particle suspension flow and biomedical such as blood flow in micropipes and drug delivery. It is known that the interactions between the particles in Non-Newtonian liquids have a profound effect on the viscosity of the liquid under shear rate region. In order to verify the effect of Non-Newtonian on the pump performance. Our interests in this work are to study the effect of the height, rotor eccentricity and dynamic parameters on the flow behavior of non-Newtonian liquid in a viscous micropump. The influence of Reynolds number on the variation of bulk velocity and stress as a function of the eccentricity and channel height will be investigated. We focus on the characterization of the changes in non-Newtonian fluids in a Micro device.

\section{VISCOSITY MEASUREMENTS}

Carboxymethyl cellulose (CMC) is a non-Newtonian liquid used in this work and it has a very wide range of application. ${ }^{16)}$ The viscosity of CMC was measured by a Haake rheometer RheoStress RS100, which is computer controlled. Samples have been tested under controlled rate (CR) mode and oscillation (OSC) mode, in the linear viscoelastic regime with the corresponding range of stress of 1-50 Pa. All measurements were performed using cone and plate geometry of diameter equal to $35 \mathrm{~mm}$. The cone angle was $4^{\circ}$ and the gap at the tip of the cone was $0.137 \mathrm{~mm}$.

\section{PROBLEM DESCRIPTION}

\subsection{Pump Geometery}

The pump geometry is shown in Figure 1. The dimensionless parameters, which are associated to the geometry, are channel height $(\mathrm{S})$ and cylinder eccentricity $(\varepsilon)$, defined as:

$\mathbf{L}$

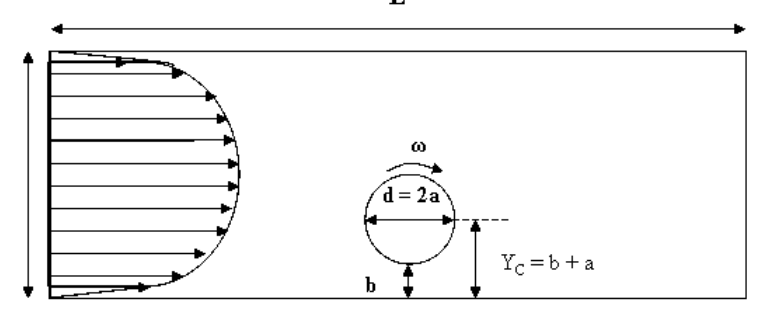

Fig. 1. Problem geometry and variable velocity distribution.

$$
\begin{aligned}
& S=\frac{h}{d} \\
& \varepsilon=\frac{\left(h-2 Y_{c}\right)}{h-d}
\end{aligned}
$$

Where $\mathrm{d}$ is the cylinder diameter and $\mathrm{h}$ is the channel height, $Y_{c}$ the distance between the lower wall of the channel and the center of the cylinder. The cylinder rotates with an angular velocity $\omega$ and is placed at different positions between the upper and lower plates of the channel based on the eccentricity. The pressure is exerted on the inlet and outlet of the channel. A higher pressure is exerted at the outlet to simulate the pressure head the pump should supply. This head is desirable to conquer the pressure drop in whatever circuit the pump is attached to. The dimensionless pressure rise is characterized by $\left(\Delta \mathrm{P}^{*}\right)$ :

$$
\nabla P^{*}=\frac{P-P_{o}}{\rho v^{2} / d^{2}}
$$

and the dimensionless bulk velocity $\left(\mathrm{u}^{*}\right)$ is defined as:

$$
u^{*}=\frac{U}{\omega a}
$$

Where $\mathrm{P}$ is the pressure at pump exit, $\mathrm{P}_{0}$ the pressure at pump inlet, $v$ the kinematic viscosity, $\rho$ fluid density and $\mathrm{U}$ the average velocity of the Bingham plastic.

\subsection{Mathematical Equations and Boundary Conditions}

For incompressible and steady state non-Newtonian fluid, the continuity equation and the equation of motion are:

$$
\begin{aligned}
& \nabla \cdot V=0 \\
& (V \cdot \nabla V)+\nabla p+\nabla \cdot \tau=0
\end{aligned}
$$

With :

$$
\nabla \cdot \tau=-\nabla \cdot(\eta \dot{\gamma})
$$

Where $\tau$ is the extra stress tensor, $\dot{\gamma}$ the rate of strain tensor, $V$ the tangential velocity and $\eta$ the fluid viscosity. Since nonNewtonian fluid respond to the imposed flows. A different constitutive equation is needed, $\tau=f(\nabla V, V$ material information).

The main independent parameters in the solution are the channel height $\mathrm{S}$, the Reynolds number Re and the eccentricity $\varepsilon$. The flow will be assumed laminar, incompressible and steady state, and the fluid is non-Newtonian. 
The boundary conditions are applied on a two-Dimensional channel with a symmetrical wall sides. The flow is assumed to be homogeneous and no slip boundary conditions are used. The velocities at the upper and lower walls are zero. The rotating cylinder with angular velocity $\omega$ causes the movement of the flow. The pressure is specified on the inlet of the micropump. The physical properties used in constitutive equations, $\eta, \rho$ are obtained from experimental measurements. The validity of calculation results has been checked by comparing with the previous experiment (see Fig.10).

\section{NUMERICAL SOLUTION}

As it is well known, the range of validity of the Newtonian constitutive equation is limited to some kind of liquid. However, the flow phenomena observed with non-Newtonian fluids cannot be predicted by the classical Navier-Stokes equation. Non-Newtonian behavior has many facets. One of them is the shear rate dependence of the shear viscosity. Therefore, we need to propose a model to describe the flow behavior of non-Newtonian fluid.

The CFD package POLYFLOW 3.92 (FLUENT COMPANY) is used to solve Navier-Stokes equations and the proposed model. This CFD package uses the finite volume method. It enables the use of different discretization schemes and solution algorithms, together with various types of boundary conditions. As part of the same package, (a preprocessor) Gambit is used to draw the geometry and generate the required grid for the solver. An unstructured grid with triangle elements is used. The upper and lower walls were divided into non-uniformly spaced elements using pave meshing scheme with a size 0.1 . The size specifies whether the nodes will be denser at the center of the edges or at its ends, and also specifies the intensity of this distribution. In addition to this meshing method, grid adaptation by the solver itself was performed in the gap between the cylinder and the lower wall for cases of high eccentricities, where this gap size is very small. The grid was finer in regions near the center and was coarser in regions far upstream and downstream as shown in Fig. 2. Different meshes were used at the beginning to determine the optimum grid size, four meshes have been tested for the case of $\mathrm{s}=3.5, \varepsilon=0.875$. The sizes of all four grids are shown in Table I. As a convergence criterion in the present work, the solver iterated the equations until it stabilized at a constant value. Grid independent solution was assured by observing three parameters. The first parameter is the distribution of the $\mathrm{x}$-velocity component on a vertical plane just one diameter from the cylinder axis. The second parameter is the shear stress. The third parameter is the average velocity of the flow on the outlet from the pump, which will give a good indication of the effect of the grid size in the entire micro-pump domain.

\subsection{Optimization and Evolution}

The idea of optimization provides a significant savings in computational time. Therefore, optimization suggests that for a given set of possible models, there exists a model that is the best fit to the experimental results. Non-Newtonian flow has been characterized by different models: Carreau-Yasuda, Modified Herschel-Bulkley and Modified Bingham, given by equations 9, 10 and 11 respectively. Modified Bingham model proposed an alternative model to describe the flow of CMC fluid. This model provides a better fit as shown in Figure 3; the decrease of viscosity with shear rate is called shear thinning. It is due to the deformation and break down of the structured material. At some point well down the viscosity curve, we see the beginnings of a flattening out, and if data at a high-enough shear rate or shear stress is available, then a second constant viscosity region, $\eta_{\infty}$, is usually seen. Thus we have the two limiting Newtonian viscosities, $\eta_{0}$ and $\eta_{\infty}$ separated by a Modified Bingham Model.

Carreau-Yasuda:

$$
\eta=\eta_{\infty}+\left(\eta_{0}-\eta_{\infty}\right)\left[1+(\lambda \dot{\gamma})^{a}\right]^{n-1}
$$

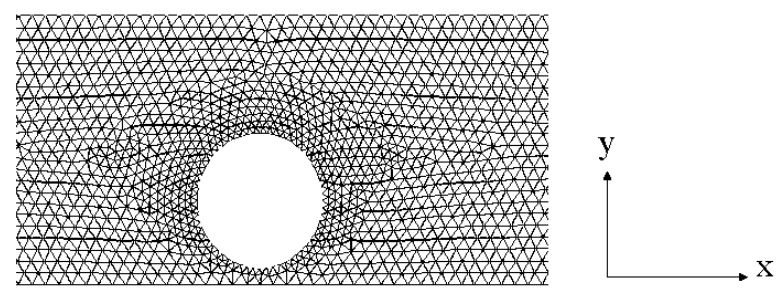

Fig. 2. An example of mesh generation.

Table I. Description of the finite element grids for the case of $\mathrm{s}=2.5$ and $\varepsilon=1.125$, are given the number of elements, nodes and dimensionless bulk velocity.

\begin{tabular}{|l|l|l|l|}
\hline $\begin{array}{l}\text { Number of cylinder } \\
\text { element X number } \\
\text { of wall elements }\end{array}$ & $\begin{array}{l}\text { Number } \\
\text { of nodes }\end{array}$ & $\begin{array}{l}\text { Total } \\
\text { number of } \\
\text { elements }\end{array}$ & $\mathbf{u}^{*}$ \\
\hline $60 \times 200$ & 5740 & 11020 & 0.00232757 \\
\hline $80 \times 210$ & 6849 & 13198 & 0.002272604 \\
\hline $90 \times 280$ & 11572 & 22494 & 0.00220069 \\
\hline $95 \times 330$ & 14988 & 29221 & 0.002264512 \\
\hline
\end{tabular}


Modified Herschel-Bulkley:

$$
\eta=\tau_{0}\left(\frac{1-\exp \left(\frac{-3 \dot{\gamma}}{\dot{\gamma}_{c}}\right)}{\dot{\gamma}}\right)+k\left(\frac{\dot{\gamma}}{\dot{\gamma}_{c}}\right)^{n-1}
$$

Modified Bingham Model:

$$
\eta=\eta_{0}+\tau_{0}\left(\frac{1-\exp (-m \dot{\gamma})}{\dot{\gamma}}\right), \quad m=\frac{3}{\dot{\gamma}_{c}}
$$

Where $\eta_{\mathrm{o}}=8.3$ Pas, zero-shear rate viscosity, $\tau_{\mathrm{o}}=301 \mathrm{~Pa}$, yield stress, and $\mathrm{m}=0.4398$ are the material properties have been attained from the modified Bingham model, $\dot{\gamma}_{c}$ the critical rate of strain, $\mathrm{k}$ the consistency factor, $\lambda$ the natural time, a the index that controls the transition from the Newtonian plateau to the power-law region and $n$ the power-law index. Papanastasiou ${ }^{12)}$ proposed a modification in the Bingham equation by introducing a material parameter, which controls the exponential growth of stress. Papanastasiou 's modification, when applied to the Bingham model, becomes

$$
\tau=\tau[1-\exp (-m \dot{\gamma})]+\eta \dot{\gamma},
$$

Where $\mathrm{m}$ is the stress growth exponent and $\tau_{\mathrm{y}}$ the yield stress. As shown in Fig. 4, the exponential modification has now been a standard way to treat viscoplastic materials, and a surplus of papers has appeared in the literature, applying this model to various types of flows. ${ }^{10,11)}$

The rate of strain dependence of the viscosity causes nonlinearity, it often makes computations difficult. There are parameters as velocity and pressure consequently are responsible for the non-linearity. Therefore, we expect to reach the solution by assuming an evolution function $(\mathrm{f}(\mathrm{s}))$ :

$$
f(L)=L, \quad 0<L<1 \quad, \Delta \mathrm{L}=0.01
$$

Polyflow calculates a solution at a discrete set of $\mathrm{L}_{\mathrm{n}}$, defined by

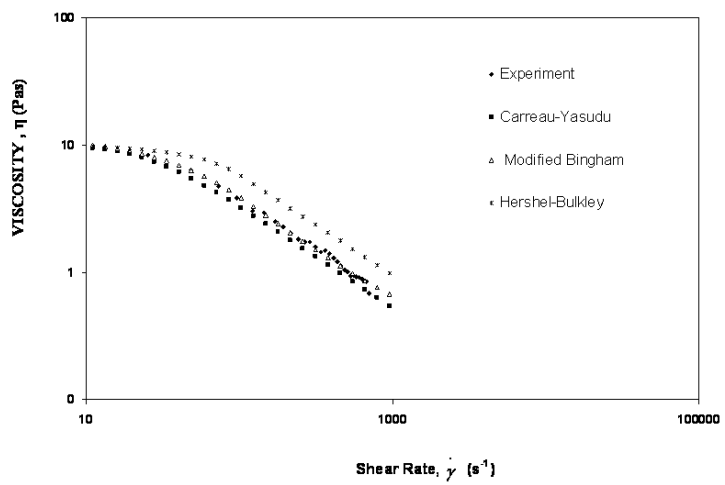

Fig. 3. Viscosity variation as a function of shear rate using different models.

$$
\begin{aligned}
& \mathrm{X}_{\mathrm{n}}=\mathrm{X}\left(\mathrm{L}_{\mathrm{n}}\right) \\
& \mathrm{L}_{\mathrm{n}}=\mathrm{L}_{\mathrm{n}-1}+\Delta L_{\mathrm{n}}
\end{aligned}
$$

Where $\mathrm{X}$ is the vector of nodal unknowns such as velocity. Where the subscript $\mathrm{n}$ refers to the $\mathrm{L}$ step. The next iteration $\mathrm{X}_{\mathrm{n}+1}$ is calculated by:

$$
\mathrm{X}_{\mathrm{n}+1}=\mathrm{X}_{\mathrm{n}}+\Delta \mathrm{L}_{\mathrm{n}} \mathrm{X}_{\mathrm{n}}
$$

If the solution converges, the computation will continue.

\section{RESULTS AND DISCUSSION}

\subsection{The effect of rotor eccentricity}

The rotor eccentricity has a crucial effect on the performance of micro viscous pump. It is the cylinder eccentricity that provides the driving force to the fluid inside the micropump channel, by introducing unequal shear stresses on the upper and lower surfaces of the cylinder. For Non-Newtonian fluid, the shear stress increases with increasing the rate of strain. Figures 5 and 6 show the curves $\mathrm{u}^{*}=f(\varepsilon)$ and $\tau=f(\varepsilon)$ respectively, where $\mathrm{S}$ (channel height) is maintained constant at 3.5. It is clear that $\mathrm{u}^{*}$ is insignificant with increasing the eccentricity at different $\mathrm{p}^{*}$ investigated. However, the bulk velocity start to decrease sharply in the range of $\varepsilon>1$. It can be seen that the stress generated by the flowing fluid is starting to decrease with increasing the eccentricity more than 0.9375 . Increasing the eccentricity causes a decrease in stress shearing. This is due to the gap between the rotor and the lower plate is very small which causes a decrease in the shearing between the fluid particles and the wall. On the other hand, low shear is not enough to break down the agglomeration between the particles and subsequent a decrease in the bulk velocity.

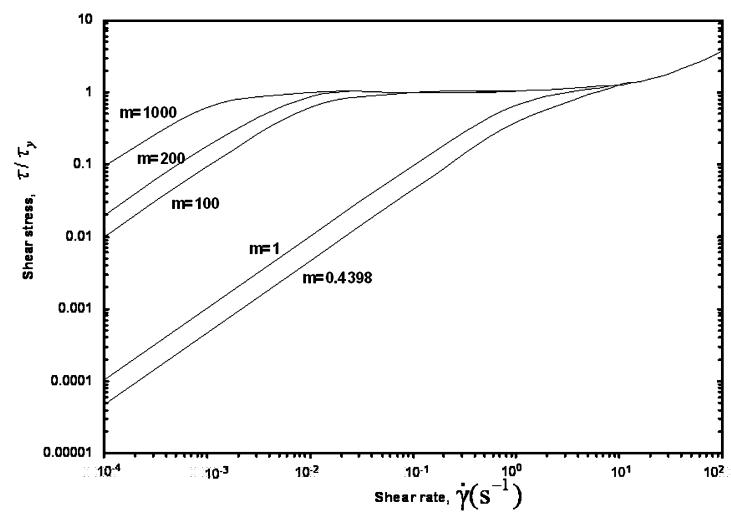

Fig. 4. Shear stress vs. shear rate according to the modified Bingham equation for several values of the stress growth exponent $\mathrm{m}$. 
Increasing pressure will cause dissipation between the particles, therefore the particles movement will be faster and the shearing between the particles and between the particles and the wall will rise. It can be concluded that the stress shearing and the bulk velocity decreases with increasing the eccentricity at $\varepsilon>0.9375$ and low Reynolds number. An increase in the gradient pressure increases the stress and the bulk velocity.

\subsection{Effect of Reynolds number}

In this problem, the average velocity in the channel $\bar{u}=\frac{1}{h} \int u d y$ is an output of the solution. Reynolds number will be defined as: $\operatorname{Re}=\frac{\omega d^{2}}{2 v}$. The Reynolds number for micropump is based on the rotor angular velocity, and not on the average fluid velocity in the micropump. The calculation of the flow of a Newtonian fluid for $\mathrm{Re}=1,10,50$ and 100 has been solved in the literature. ${ }^{17)}$ It has been noted that higher Reynolds numbers means that the effect of the viscous forces is less significant when compared to the inertia forces. Also, previous studies showed that the pumping efficiency of a Newtonian fluid decreased one order of magnitude when Reynolds number increased from $\operatorname{Re}=1$ to $\operatorname{Re}=10$, and it decreased another order

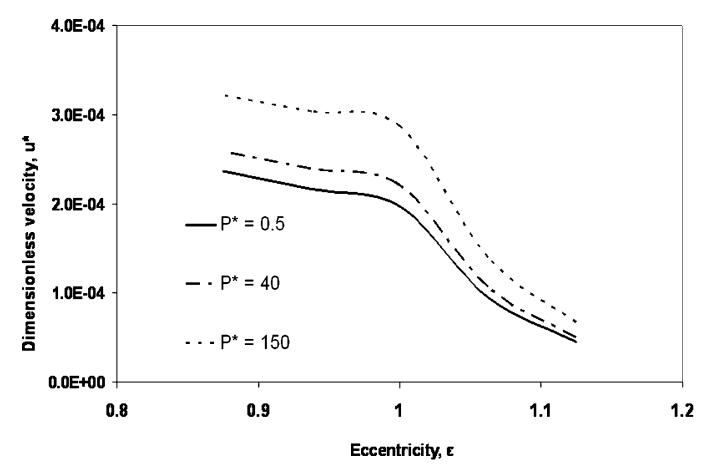

Fig. 5. Changes of the bulk velocity as a function of eccentricity. For $\mathrm{P} *=0.5,40$ and 150 .

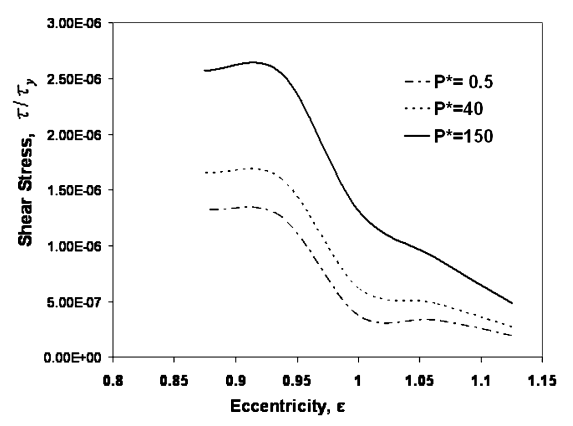

Fig. 6. Changes of the stress as a function of eccentricity. For $\mathrm{P}^{*}=0.5$, 40 and 150 . of magnitude when Reynolds number was increased to $\operatorname{Re}=100 .{ }^{17}$

In Figure 7, the bulk velocity is plotted as a function of Reynolds number (Re) with different eccentricities and constant $\mathrm{p}^{*}=0.5$. We notice that the bulk velocity increases with decreasing eccentricity at constant $\mathrm{p}^{*}=0.5$. Figure 8 shows the effect of Reynolds number on the generated stress with different eccentricities. It is noticed that when $\operatorname{Re}<40$, Reynolds number has insignificant effect on the stress. For $\operatorname{Re}>40$, it is observed that the stress is increasing sharply with increasing Reynolds number. This is due to the interaction forces between the particles. These forces need high shearing generated by increasing the velocity to be broken down.

Viscosity is recognized as a material property of a liquid. Since the viscosity of non-Newtonian is not constant and it depends on the rate of strain. Therefore, it is very important to cover the changes of the viscosity in micropump. This has been demonstrated in Figure 8, the viscosity as a function of the eccentricity. It can be seen that the viscosity is increasing with increasing the eccentricity. The operating parameters are: $\operatorname{Re}=100, \varepsilon=1.125$ and $\mathrm{p}^{*}=0.5$. Narrow gap between the rotating cylinder and the lower plate of the channel leads to

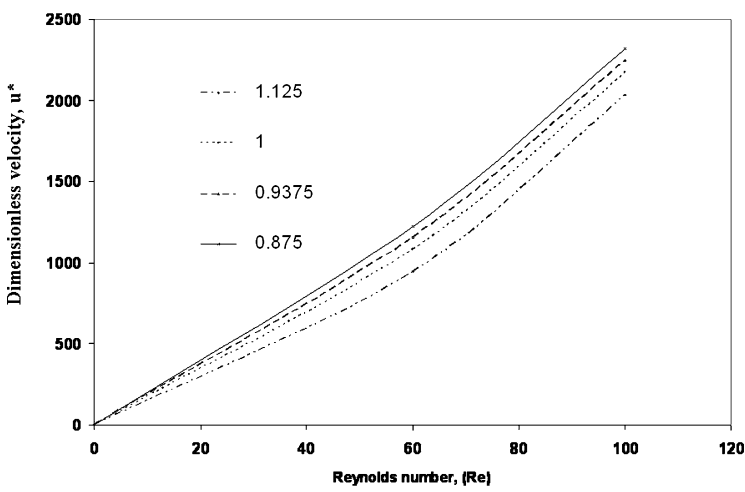

Fig. 7. Changes of the bulk velocity as a function of Reynolds number

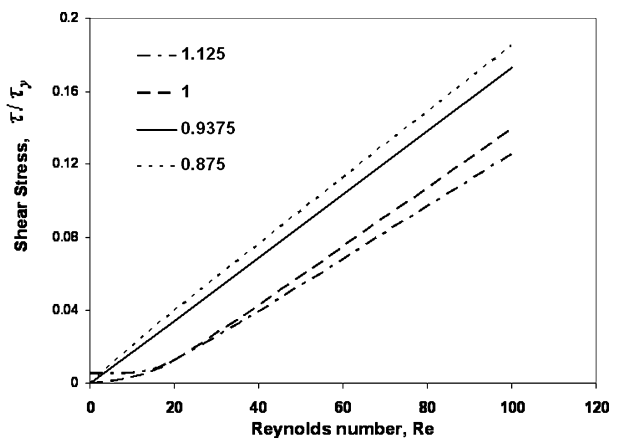

Fig. 8. Changes of the stress as a function of Reynolds number. 
increase the viscosity. This is due to a decrease in the rate of strain and the shearing stress that cause a less deformation in the fluid structure and subsequent an increase in the viscosity. Also, it can be seen that the bulk velocity is decreasing with increasing the eccentricity. It is crucial to note the effect of Reynolds number on the variation of the velocity as a function of the eccentricity. It can be concluded that the bulk velocity decreases with increasing eccentricity at high Re.

\subsection{Effect of micropump channel height}

Steady state cases were simulated. Experimental results were obtained from $^{5}$, while numerical results were obtained from. ${ }^{7,17)}$ Figure 10-b compares the results calculated by Sharatchandra et al. ${ }^{7)}$, Abdelgawad ${ }^{17)}$ and 10-a for non-newtonian. It shows the decrease in the bulk velocity of Newtonian fluid for $\mathrm{S}>1.5$. It is clear that the increase in channel height results a decrease in the velocity of Non-Newtonian fluid for $\mathrm{S}<2$. This is due to the reduction of the shear stress on the upper cylinder surface resulting from the larger distance between the cylinder and the upper wall; this reduces the flow driving force. The change in the channel height of viscous micropump affected on the resulting stress as shown in Figure 11. The working parameters are: $\varepsilon=0.9, \Delta \mathrm{P}^{*}=0.5$ and $\mathrm{Re}=0.5$. It is observed that the stress decrease with increasing the height of the channel up to a certain height then followed by an increase in the stress. The stress contours in Figure 12 are better explained in the context of Figure 11, which shows the shear stress distribution around the cylinder. Increasing the height cause agglomeration between the particles and subsequent the viscosity increase. Due to that, the shear stress and rate of strain decrease. A more interesting facet is observed that after specific height the stress increases with increasing the height. The variation in the shear stress contours is shown in Figure 12-a, b, c and d. This can be explained that the viscosity

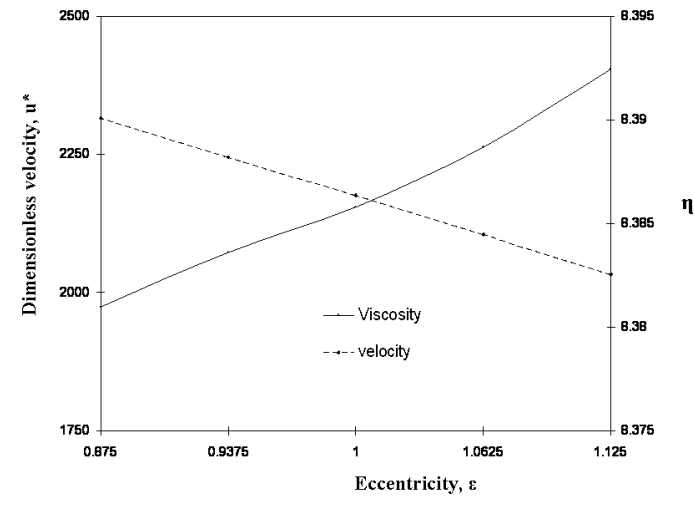

Fig. 9. Changes of the bulk velocity as a function of eccentricity at high Reynolds number. decreases with increasing the height and the agglomeration dissipate between the particles.

Figure 13 shows the influence of the variation in channel heights on the computed streamlines. The operating parameters are: $\varepsilon=1.125$ and $\mathrm{p}^{*}=1.5$. In Figure 13 -a, the flow structure reveals co-rotating vortices around the cylinder. The thinning of the vortices can be explained by decreasing the viscosity of the fluid results in an increase the rate of strain and subsequent shear stress increases as shown in Figure 13-d.

\subsection{Pump Performance}

In this section, we focus on the pump performance. The mesh affects the distribution of the computed stress and a subsequent the computed moment coefficient $\left(\mathrm{C}_{\mathrm{M}}\right)$ calculated by
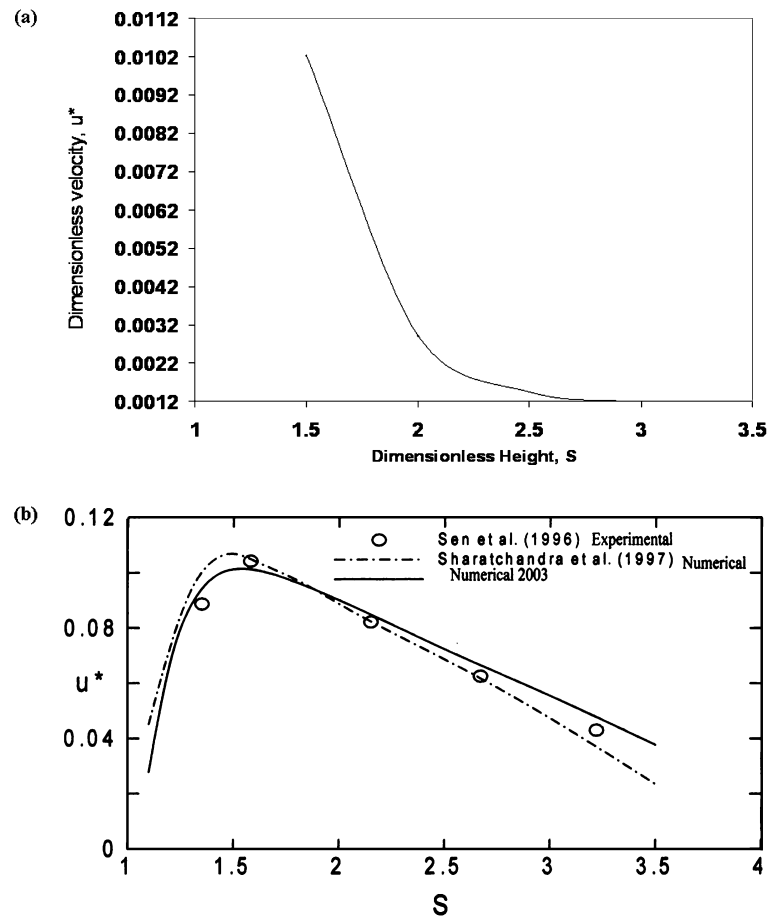

Fig. 10. Changes of the bulk velocity as a function of channels height (a) non-Newtonian (b) Newtonian. $\varepsilon=0.9, \mathrm{P} *=0.5$ and $\mathrm{Re}=0.5$

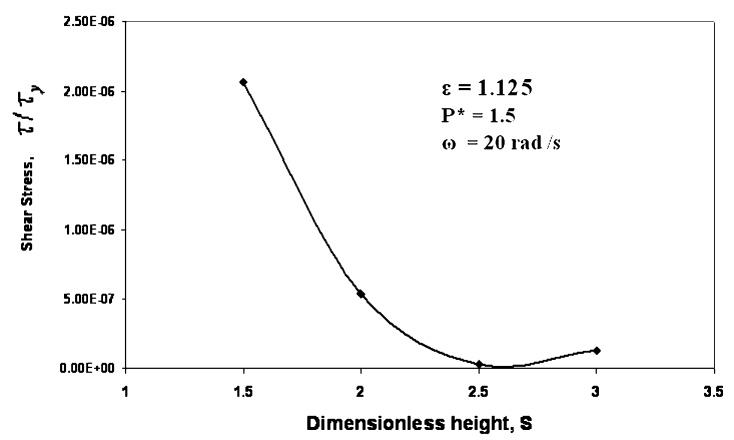

Fig. 11. Changes of the stress as a function of channel height. 


$$
C_{M}=\frac{M}{\frac{1}{2} \rho V^{2} D^{2}}
$$

Table II shows the computed moment coefficients values. A magnitude of the pumping efficiency, E, is calculated to depict the performance of the pump to be

$$
\mathrm{E}=\frac{\frac{\dot{\mathrm{m}} \mathrm{P}}{\rho}}{M \omega}
$$

In non-dimensional terms, the efficiency reduces to:

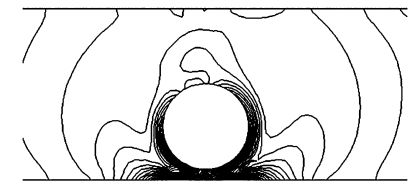

(b)

(d)

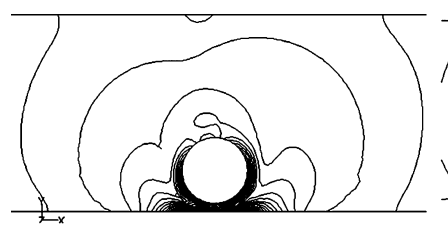

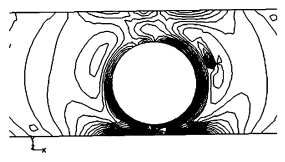

(a)
Fig. 12. Stress contours for $\varepsilon=1.125, \omega=10 \mathrm{rad} / \mathrm{s}$ and at different dimensionless heights $(\mathrm{s})$ : $\mathrm{a}-\mathrm{s}=1.5, \mathrm{~b}-\mathrm{s}=2, \mathrm{c}-\mathrm{s}=2.5, \mathrm{~d}-\mathrm{s}=3$.

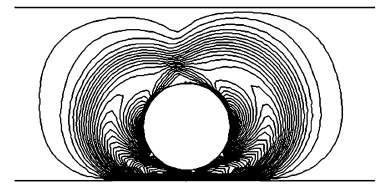

(b)

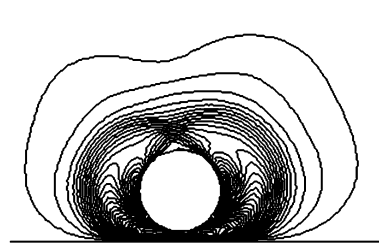

(d)

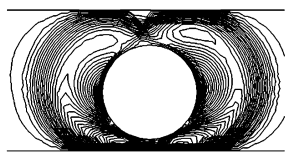

(a)

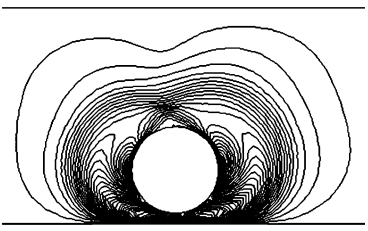

(c)
Fig. 13. Streamlines for $\varepsilon=1.125, \omega=10 \mathrm{rad} / \mathrm{s}$ and at different dimensionless heights: $\mathrm{a}-\mathrm{s}=1.5, \mathrm{~b}-\mathrm{s}=2, \mathrm{c}-\mathrm{s}=2.5, \mathrm{~d}-\mathrm{s}=3$.

Table II. The $\mathrm{C}_{\mathrm{M}}$ values at different pressures.

\begin{tabular}{|l|l|l|l|l|}
\hline$P^{*}$ & 0.5 & 1.5 & 2.5 & 40 \\
\hline$S=1.5$ & 0.1085 & 0.1085 & 0.1085 & 0.1085 \\
\hline$S=3$ & 0.8392 & 0.8392 & 0.8392 & 0.8392 \\
\hline
\end{tabular}

$$
\mathrm{E}=\frac{S \mathrm{u} * \mathrm{P}^{*}}{\mathrm{C}_{\mathrm{m}} \operatorname{Re}^{2}}
$$

It is perceptible from Figure 14 that the pump performance is associated to the channel height. The pumping performance is increasing with increasing $\mathrm{p}^{*}$, the efficiency of the pump at $\mathrm{S}=1.5$ is higher than at $\mathrm{S}=3$. This is because the flow structure will change with shearing. Since the change in the rate of strain is constant (angular velocity is constant), the variation in the shear stress will be proportional to the change in the viscosity of non-Newtonian flow. Therefore the structure will deform and cause an increase in the movement of the particles and increase in the pumping performance. It is clear that increasing the channel height reduces the velocity gradients on the cylinder surface and therefore reduces the pumping efficiency, and hence the viscous forces decrease. It is concluded that the pumping performance of non-Newtonian fluid is increasing with $\mathrm{p}^{*}$ and decreasing with the channel height.

\section{CONCLUSION}

The objective of the present study was to explore the effect of Micro pump geometry on the flow behavior of non-Newtonian fluid. Therefore, it is desirable to investigate the deformation in the structure of fluid flow system in micro pump. Numerical solution of the flow through the micropump is calculated by simulating Navier-Stokes equations. Experimental results model the effect of shear thinning using a modified Bingham model. It was found that, the stress shearing and the bulk velocity decrease with increasing the eccentricity at low Reynolds number. Increasing the channel height of the micro pump influence the flow behavior of non-Newtonian fluid. The viscous forces dominate non-Newtonian fluid flow in micropump. The changes of non-Newtonian fluid structure in

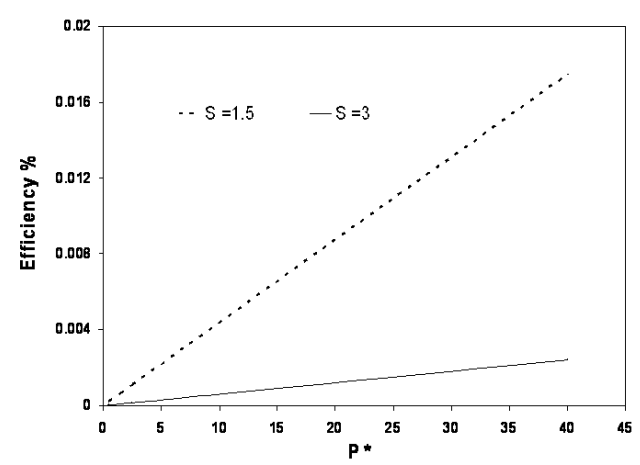

Fig. 14. Pump performance at $\mathrm{S}=1.5$ (dotted line) and $\mathrm{S}=3$. 
micropump are related to Reynolds number, eccentricity and channel height. The pumping performance of non-Newtonian fluid is increasing with pressure and decreasing with the channel height. In the future, more investigations should be conducted on non-Newtonian fluid in different designs of micropump.

\section{REFERENCES}

1) Voigt P, Schrag G, Wachutka G, A66, 9 (1998).

2) Schomburg WK, Goll C, A 64, 259 (1998).

3) Shoji S, Esashi M, J. Micromech. Microeng. 4, 157 (1994).

4) Gravesen P, Branebjerg J, Jensen OS, J. Micromech. Microeng. 3, 168 (1993)

5) Sen M, Wajerski D, Gad-el-Hak M, Journal of Fluid Engineering, 118, 624 (1996)

6) Podell GM, Kovasznay LSG, Journal of Fluid Mechanics, 50, 535 (1971).
7) Sharatchandra MC, Sen M, Gad-el-Hak M, ASME, Journal of Fluids Engineering, 119, 372 (1997).

8) Decourtye D, Sen M, Gad-El-Hak M, IJCFD, 10, 13 (1998).

9) Yoshida K, Kikuchi M, Park JH, Yokota S, J. sensors and actuators $A, 95,227$ (2002).

10) Mitsoulis E, J. Non Newtonian Fluid Mech. 105, 1 (2002).

11) Blackery J, Mitsoulis E, J. Non Newtonian Fluid Mech. 70, 59 (1997).

12) Papanastasiou TC, J. Rheol. 31, 385 (1987).

13) Piau JM, J. Non Newtonian Fluid Mech. 102, 193 (2002).

14) Liu BT, Muller SJ, Denn MM, J. Non Newtonian Fluid Mech. 102, 179 (2002)

15) Benjamin T. Liu, Susan J. Mullar, Morton M. Denn, J. Non Newtonian Fluid Mech. 113, 49 (2003).

16) Mamdouh T. Ghannam, M. Nabil Esmail, Journal Applied Polymer Sci. 64, 289 (1997).

17) Abdelgawad M, Hassan I, Esmail N, Journal of Microscale Thermophysical Engineering. Accepted. 